\title{
Assessing the Role of Knowledge, A wareness, Practice in Achieving Medication Adherence among Hypertensive Patients
}

\author{
Satish $\mathbf{S}^{1}$, Minnu Sara Sam ${ }^{2}$, A R Shabaraya ${ }^{3}$ \\ ${ }^{1}$ Associate Professor, Department of Pharmacy Practice, ${ }^{2}$ Student, PharmD, Department of Pharmacy Practice, \\ ${ }^{3}$ Professor \& Head, Department of Pharmacy Practice, \\ Srinivas College of Pharmacy, Mangalore, Karnataka-574143
}

Corresponding Author: Minnu Sara Sam

\begin{abstract}
Medication adherence is that the degree to which the person's behaviour corresponds with the agreed recommendations from a health care provider. Knowledge and the awareness concerning the hypertension and the antihypertensive medications have an important role within the medication adherence; lack of this can have negative impact on the medication adherence.

The assessment of medication adherence in the study was meted out by using Morisky Scale. It's a cross sectional study conducted in 150 individuals in Kankanady, Valachil, Valencia, Jeppu, Padil of Mangalore in Karnataka. Patient data collection forms were used to collect the information. Present study reflected that patients had knowledge regarding normal BP, symptoms and complications; however majority thinks hypertension can be curable. A positive attitude and practice were observed among the patients apart from practice of normal exercise. Analysing the data using MAMS-8 scale, study showed only $11.3 \%$ of the population were found to be high adherent that shows the decrease in the adherence rates. 34\% had moderate adherence and the remaining 54\% were low adherent. Assessment of data suggests that majority lack complete information about hypertension and its management.

The study results revealed that hypertension related knowledge, awareness and practice plays a serious role in improving medication adherence.
\end{abstract}

Keywords: Medication adherence, Hypertension, Knowledge, Awareness, Morisky scale.

\section{INTRODUCTION}

Medication-taking behaviour is extremely complex and individual, requiring numerous multi-factorial strategies to boost adherence. Patients ought to understand the importance of taking their pill, refilling their prescriptions and getting well. [1] Hypertension is a condition within which the pressure of the blood is chronically elevated. ${ }^{[2-4]}$

Hypertension can lead to cardiovascular morbidity and mortality ensuing from target-organ damage to blood vessels in the heart, brain, kidney and eyes [5] It has also been recognized that adherence to Antihypertensive drugs (AHD) is an important mediator of achieving desirable BP management, however poor adherence with medication still remains a problem among hypertensive patients. It's been known as one of the main causes of failure in achieving blood pressure control. [6] However, non-adherence shows larger risk in older people which finally results in poor disease control. ${ }^{[7-8]}$

The best known and most generally used scales for analysing adherence is the medication adherence questionnaire (MAQ) by Morisky et al which has several advantages. It's the simple and effective 
tool to deal with non-adherence in patients. Morisky medication adherence scales are the foremost accepted self-reported medication adherence measures, recommended to serve as screening tools in a very clinical setting. ${ }^{[9]}$ It's proved to be a vital resource to address adherence concerns, such as lack of knowledge on hypertension and the antihypertensive medication.

Knowledge and awareness regarding their disease status and their diagnosis, target, and recent blood pressure values aid to improve the outcome of patients. Knowledge positively influences the adherence and therefore the good adherence is associated with the good blood pressure control. Patient should be received adequate information about therapeutic methods and the consequences of failure to treat or improper treatment of their disease. Morisky is also the shortest and easiest to score and very adaptable for various groups of medications. If a patient scores higher on the scale, they're evaluated to be more adherent to the antihypertensives. Based on the patient score on the Morisky scale, the clinician and the health care professionals can identify underlying issues that prevent patients from taking their medications properly, if at all. Advantage of the Morisky tool lies in its ease of use and also the cost effectiveness. Clinicians have no burden in conducting test whereas the patients are asked to put in minimal effort to complete the short questionnaire.

\section{MATERIALS \& METHODS}

Study Site: The study was conducted in 150 individuals in Kankanady, Valachil, Valencia, Jeppu, Padil of Mangalore in Karnataka.

Study Design: A prospective cross sectional study to assess the role of knowledge, awareness, practice in achieving medication adherence among the hypertensive patients.

Sample Size: A total of 150 patients were included who met the study criteria (inclusion and exclusion criteria).
Study Duration: The study was conducted for a period of 6 months from September 2019 to March 2020.

Ethical Approval: Ethical clearance was obtained from the Institutional Ethics Committee (IEC), Srinivas Institute of Medical Sciences and Research Centre, Mukka, Mangaluru, India (Ref no. 2019/10/2820/1)

Study Subjects: The study includes 150 Patients diagnosed with hypertension and on oral antihypertensive agents who met the subsequent study criteria.

\section{Study criteria:}

\section{- Inclusion criteria:}

Patients of age 31-70years of either sex with hypertension

Patients on oral antihypertensive agents.

Patients who are willing to participate in the study

\section{- Exclusion criteria:}

Patients with visual and hearing impairment Pregnant and lactating women

Terminally ill patients

Patients <18years or>70years

Patients who did not agree to participate in the study

\section{Source of data:}

Data(s) for the study were collected using data collection form subjects. Area wise they were distributed from different parts of Dakshina Kannada district. The data was collected through direct interaction with the patients.

\section{Study method:}

Preparation for the study: Patient data collection forms were prepared and it was used for collecting data from patients.

Patient selection: Patients were segregated based on inclusion and exclusion criteria.

\section{Obtaining informed consent (ICF)}

Inform consent form was prepared in Kannada and English and the same was used. Before selection of subjects, the consent form was orally explained to the 
participants before filling it and made them understood. In the study only the participants willed to fill ICF were included.

\section{Patient categorization:}

$\operatorname{Data}(\mathrm{S})$ were collected using data collection form and Morisky medication adherence scale (mmas-8) for the assessment of medication adherence and later it was correlated to categorize patients into highly adherent, medium adherent and low adherent based on 8 item MMAS and it will be reported and recorded.

Statistical Analysis: Statistical analysis involves collecting and scrutinizing each data sample in a set of items from which samples can be drawn and were analysed using Microsoft Excel.

\section{RESULT \\ DEMOGRAPHIC CHARACTERISTICS OF STUDY POPULATION:}

A total of 150 patients participated in our study. $45.3 \%$ of participants were males and $54.6 \%$ of patients were females. The highest percentage of age group was $51-70$ years $(76.6 \%)$ followed by $71-90$ years $(16 \%)$ and age group of 31-50 years $(7.3 \%)$.

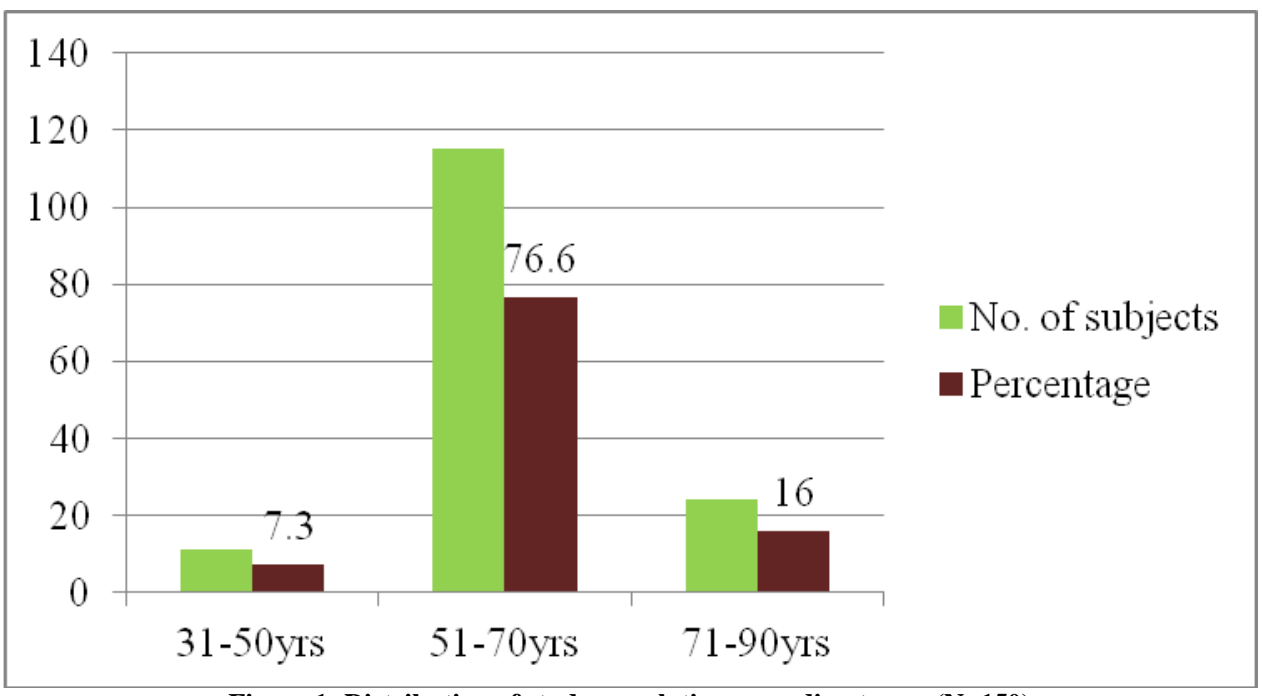

Figure 1: Distribution of study population according to age ( $\mathrm{N}=150)$

AREA-WISE DISTRIBUTION:

Area-wise distribution of subjects from different parts of Dakshina Kannada (D.K.) district participated within the study is as follows (Table 1):

Table 1: Area-wise distribution of subjects $(\mathrm{N}=150)$

\begin{tabular}{|l|l|}
\hline Area (D.K. district) & No. of patient \\
\hline Kankanady & 39 \\
\hline Valachil & 26 \\
\hline Valencia & 31 \\
\hline Jeppu & 23 \\
\hline Padil & 31 \\
\hline
\end{tabular}

\section{RESONSE TO PATIENT'S KNOWLEDGE ASSESSMENT OF HYPERTENSION:}

The knowledge of the participants regarding hypertension was assessed using a questionnaire that was validated. $70.6 \%$ of the population had good knowledge of hypertension. Around $71.8 \%$ of the population knew the values of normal blood pressure, $86.4 \%$ of them had known that hypertension could progress along with age and that risk of developing hypertension was high if there is family history of hypertension. $80.6 \%$ of the subjects know that it's a treatable condition. They are also aware about the risk factors of hypertension like aging (83.5\%), smoking (43.7\%), eating fatty foods $(46.6 \%)$ and overweight $(39.8 \%) .82 .5 \%$ of the subjects believe that regular physical exercise can control blood pressure and a majority also knows that salt consumption could lead to increase in BP. 
Satish $S$ et.al. Assessing the role of knowledge, awareness, practice in achieving medication adherence among hypertensive patients.

But $60.2 \%$ think that medication alone can control BP.

Table 2: Response To Patient's Knowledge Assessment Of Hypertension

\begin{tabular}{|l|l|l|l|l|}
\hline Questions & Yes & No & $\begin{array}{l}\text { Yes } \\
(\mathbf{\%})\end{array}$ & $\begin{array}{l}\text { No } \\
(\boldsymbol{\%})\end{array}$ \\
\hline Knowing normal values of BP & 111 & 39 & 71.8 & 28.2 \\
\hline Increase in BP > 140/90mmHg called HTN & 98 & 52 & 66 & 34 \\
\hline HTN can progress along with the age & 124 & 26 & 86.4 & 13.6 \\
\hline Both sexes have equal chance of developing HTN & 105 & 45 & 74.8 & 25.2 \\
\hline HTN is a treatable condition & 122 & 28 & 80.6 & 19.4 \\
\hline Risk of developing HTN if family history of HTN & 132 & 18 & 86.4 & 13.6 \\
\hline Aging is greater risk of HTN & 120 & 30 & 83.5 & 16.5 \\
\hline Smoking is a risk factor for HTN & 69 & 81 & 43.7 & 56.3 \\
\hline Eating fatty foods is a risk factor for HTN & 75 & 75 & 46.6 & 53.4 \\
\hline Overweight is a risk factor for HTN & 70 & 80 & 39.8 & 60.2 \\
\hline Regular physical exercise reduces HTN & 124 & 26 & 82.5 & 17.5 \\
\hline More salt consumption increases BP & 137 & 13 & 91.3 & 8.7 \\
\hline Medication is alone in controlling HTN & 92 & 58 & 60.2 & 39.8 \\
\hline HTN can lead to life-threatening condition & 119 & 31 & 75.7 & 24.3 \\
\hline
\end{tabular}

\section{RESPONSE TO PATIENTS' \\ AWARENESS ASSESSMENT OF HYPERTENSION:}

Assessment of awareness regarding hypertension was also carried out using a validated questionnaire. The results yielded showed that $76.2 \%$ were aware of hypertension. All of them knew about having hypertension; $63.3 \%$ were aware of their target personal BP; $84 \%$ were aware that controlling blood pressure could reduce complications; $66 \%$ knew that hypertension if uncontrolled could lead to organ damage; most of them checked their BP regularly and $75.3 \%$ knew their BP values at their recent visit.

But from the present study it was observed that $68.6 \%$ think that $\mathrm{BP}$ is a curable condition and not a manageable condition. $83.3 \%$ are aware that lifestyle modifications could help control BP.

Table 3: Response To Patients' Awareness Assessment Of Hypertension

\begin{tabular}{|c|c|c|c|c|}
\hline Questions & Yes & No & $\begin{array}{l}\text { Yes } \\
\%\end{array}$ & $\begin{array}{l}\text { No } \\
\%\end{array}$ \\
\hline Knowing about having hypertension & 150 & 0 & 100 & 0 \\
\hline Knowing blood pressure values in diagnosing as hypertension & 114 & 36 & 76 & 24 \\
\hline Knowing the values of target personal blood pressure & 95 & 55 & 63.3 & 36.6 \\
\hline Controlling $\quad$ of $\quad$ blood $\quad$ pressure reduces your complications & 126 & 24 & 84 & 16 \\
\hline Uncontrolled hypertension can lead to your organ's damage & 99 & 51 & 66 & 34 \\
\hline Knowing values of blood pressure at recent visit & 113 & 37 & 75.3 & 24.6 \\
\hline Thinking that HTN is curable condition & 103 & 47 & 68.6 & 31.3 \\
\hline Changing your lifestyle helps to lower your blood pressure & 125 & 25 & 83.3 & 16.6 \\
\hline Improvement of your blood pressure over the last 12 months & 105 & 45 & 70 & 30 \\
\hline
\end{tabular}

\section{MORISKY MEDICATION ADHERENCE SCALE (MMAS-8):}

Table 4: Morisky Medication Adherence Scale (MMAS-8)

\begin{tabular}{|c|c|c|c|c|}
\hline Questions & Yes & No & Yes & No \\
\hline Do you sometimes forget to take you medication? & 68 & 82 & 45.3 & 54.7 \\
\hline Over the past two weeks, were there any days when you did not take your medication? & 36 & 114 & 24 & 76 \\
\hline Have you ever stopped taking medication without telling doctor? & 26 & 124 & 17.3 & 82.6 \\
\hline When you leave/ travel home, do you sometimes forget to take your medication & 75 & 75 & 50 & 50 \\
\hline Did you take your medicine yesterday? & 136 & 14 & 90.6 & 9.3 \\
\hline When you feel like your health is under control, do you sometimes stop your medication? & 31 & 119 & 20.6 & 79.3 \\
\hline $\begin{array}{l}\text { Taking tablets everyday is really unconvincing for some people. Do you ever feel hassled about sticking to } \\
\text { your treatment plan? }\end{array}$ & 102 & 48 & 68 & 32 \\
\hline \multicolumn{5}{|l|}{ How often you have difficulty remembering to take medicine? } \\
\hline Never & 24 & 126 & 16 & 84 \\
\hline Rarely Sometimes Often & 62 & 88 & 41.3 & 58.6 \\
\hline \multirow[t]{3}{*}{ Always } & 34 & 116 & 22.6 & 77.3 \\
\hline & 27 & 123 & 18 & 82 \\
\hline & 3 & 147 & 2 & 98 \\
\hline
\end{tabular}


The Morisky Medication Adherence Scale (MMAS-8) remains one amongst the foremost wide used mechanisms to assess patient adherence. The questions 1-7 within the Morisky scale needs dichotomous reply from the patient and address reasons for medication non-adherence. $8^{\text {th }}$ question is answered using a 5-point Likert scale regarding how often an individual has difficulty in taking medications. Scores are summed and range from 0-8.

\section{IMPACT ON MEDICATION ADHERENCE:}

From the present study it was found that the role of knowledge, awareness and the practice among the patients plays an important role in the management of hypertension and also, it was found that only $11.3 \%$ of the population was found to be high adherent which significantly shows the decrease in the adherence rates. 34\% had moderately adherence and the remaining $54 \%$ were low adherent

Table 5: Category of medication adherence using Morisky scale:

\begin{tabular}{|l|l|}
\hline Category & No. Of patients \\
\hline High Adherence $(=8)$ & $17(11.3 \%)$ \\
\hline Medium Adherence $(6-<8)$ & $52(34 \%)$ \\
\hline Low Adherence $(<6)$ & $81(54 \%)$ \\
\hline
\end{tabular}

\section{DISCUSSION}

Adherence to appropriate medical therapy for hypertension may end up in controlled blood pressure and reduction in adverse outcomes. With increasing want for long-term adherence to treatment, a reliable and valid measure of patient adherence that can be easily administered is needed. When appropriate, tailored interventions can be implemented, like education of the patient regarding hypertension care, correcting misunderstandings and incorrect beliefs regarding hypertension treatment, reducing stress and improving coping skills among patients, or establishing a treatment regimen to foster medication adherence.

Present study reflected that minor percentage had moderate level of knowledge among hypertensive patients relating to normal $\mathrm{BP}$, symptoms and complications, but majority thinks hypertension can be curable. A positive attitude and practice were observed among the patients aside from practice of regular exercise. Hence, emphasize on awareness regarding symptoms, complications and importance of physical activity should be improved significantly among male patients.

In our study, MMAS-8 was used to assess the medication adherence of the subjects. The Morisky Medication Adherence Scale, otherwise referred to as the Morisky Scale (MMAS-8), has proved to be a valuable resource to address adherence issues, such as forgetting to take medications or discontinuing medications without guidance. If a patient scores higher on the scale, they're evaluated as more adherent. If they score lower on the scale, they're presumed to be struggling with nonadherence. By understanding how the patient scored on the scale, clinicians and health organizations can identify underlying adherence concerns, that prevent patients from taking their medications correctly, if at all. The study shown that it had been found that only $11.3 \%$ of the population was found to be high adherent which significantly shows the decrease within the adherence rates. 34\% had moderately adherence and also the remaining 54\% were low adherent.

A clinical based study by Sahoo et.al., that was conducted in two health centres in Singur block, West Bengal included a total of 186 study subjects. As per MMAS score, $55.4 \%$ were highly adherent and $32.3 \%$ and $12.3 \%$ belong to the class of medium and low adherence respectively.

The classification shown from this study implies the necessity for patient education to increase knowledge regarding hypertension treatment and for effective communication between the physician and patients to enhance understanding regarding hypertension and its treatment. Study data suggest the worth of a management oriented approach to avoiding early discontinuation and improvement of the quality of regimen 
execution by helping patients to integrate their dosing into daily routines.

\section{CONCLUSION}

The study results revealed that hypertension related knowledge plays a major role in improving medication adherence. The knowledge about hypertension among the subject was found to be low. Findings from Our study can set the stage for future studies to address the causal relation between adherence and knowledge attitude and practice.

\section{Acknowledgement: None}

\section{Conflict of Interest: None}

\section{Source of Funding: None}

\section{Ethical Approval: Approved}

\section{REFERENCES}

1. Wick Jy. Rx Focus. Adherence Issues in Elderly Patients. Pharmacy Times. 2011; (1):32.

2. Salvi P. Hypertension and Spurious Systolic Hypertension in Youth. High Blood Pressure \& Cardiovascular Prevention. 2007;14(3):145-196

3. Gosmanova EO, Molnar MZ, Alrifai A, et al., Impact of non-adherence on renal and cardiovascular outcomes in US veterans. American journal of nephrology. 2015; 42(2):151-157.

4. Beevers DG, Robertson JI, Lip G, et al., A short history of the study of hypertension. Comprehensive Hypertension. 2007: 3-20.
5. Hughes CM. Medication non-adherence in the elderly. Drugs \& aging. 2004; 21(12):793-811.

6. Shruthi R, Jyothi R, Pundarikaksha HP, et al., A study of medication compliance in geriatric patients with chronic illnesses at a tertiary care hospital. Journal of clinical and diagnostic research: JCDR. 2016; 10(12).

7. Bartosz uchmanowicz, Ewa A. Jankowska, Izabella Uchmanowicz et at., Self-Reported Medication Adherence Measured With Morisky Medication Adherence Scales And Its Determinants In Hypertensive Patients Aged $\geq 60$ Years: A Systematic Review And Meta Analysis: front pharmacol. 2019; 10(168)

8. Lee GK, Wang HH, Liu KQ, Cheung Y, et al., Determinants of medication adherence to antihypertensive medications among a Chinese population using Morisky Medication Adherence Scale. PloS one. 2013;8(4)

9. Conn VS, Hafdahl AR, Cooper PS, et al., Interventions to Improve Medication Adherence among Older Adults: MetaAnalysis of Adherence Outcomes among Randomized Controlled Trials, the Gerontologist. 2009; 49(4):447-462

10. Kocurek B. Promoting medication adherence in older adults. Diabetes Spectrum. 2009; 22(2):80-84.

How to cite this article: Satish S, Sam MS, Shabaraya AR. Assessing the role of knowledge, awareness, practice in achieving medication adherence among hypertensive patients. International Journal of Research and Review. 2021; 8(5): 324-329. DOI: https://doi. org/10.52403/ijrr.20210541 